\title{
Besprechungen
}

von

\section{Wilhelm Roux.}

\section{Greil, ALfred, Tafeln zum Vergleiche der Entstehung der Wirbel- tierembryonen. Jena 1914. gr. $4^{0} .379 \mathrm{~S} .15$ Tafeln.}

Dieses deskriptive Werk ist hier nur insoweit zu berücksichtigen, als der Autor auch kansale Folgerungen ableitet. Das geschieht sehr reichlich, indes ohne Berücksichtigung der Schranken, die der vergleichenden Forschung in dieser Hinsicht gesetzt sind, die ich in den Programmschriften der kausalanalytischen experimentellen Erforschnng der Ontogenese dargelegt habe. Die vielen kausalen Ableitungen des Autors finden teils unter direkter Verwerfung, teils unter Übergehung der experimentellen entwicklnngsmechanischen Ergebnisse statt; sie sind spezielle Anwendungen der allgemeinen theoretischen Auffassungen des Autors, welche wir bereits in Bd. 35, S. 315, charakterisiert haben; seine Auffassungen werden durch diese Beispiele noch deutlicher. Ich ersuche, der Kürze halber, die Beurteilang dort nachzulesen.

Es ist alles das, leider meist Abfällige, was wir zu sagen hatten, aufrecht zu erhalten. Seine Mißdeutungen unserer Termini und Begriffe schließen eine Verständignng aus.

Es seien einige, Greils Auffassung charakterisierende Äußerungen reproduziert.

Wesentlich vom >Ringen \& der wachsenden und sich vermehrenden Zellen and der wachsenden Zellgruppen untereinander leitet ' $G$. die Formenbildungen des Chordonier ab; er arbeitet also mit den smechanischen Massenkorrelationen* meiner Distinktion und mit Kampf der Teile; das ist nicht neu. Diese Korrelationen folgert er aus deskriptiven: vergleichend-embryologisehen Tatsachen und kommt dabei mit für ihn zwingender Notwendigkeit zu dem Urteil, daß es die von vielen kausalen Forschern durch Isolation direkt erwiesene Selbstdifferenzierung von Teilen nicht geben kann. Er sagt S. 367: »Die strenge Abhängigkeit der einzelnen sich stetig vermehrenden, in nngleichem und beengtem Wachstum sich sondernden Glieder und Formationen kennzeichnet also das zellenstaatliche Bauen und Erwerben. Daher ist es vollkommen ausgeschlossen, daß in diesem Ringen der Teile durch, Selbstdifferenzierung' zellenstaatliche Formenmannigfaltigkeit entstehe. Jede Gruppierung und Sonderung der Zellen und Zellenkomplexe, jede Ablenknng und Konzentration des Wachstums entsteht aus der einfachen Ausgangssituation in strenger gegenseitiger Abhängigkeit der Abkömmlinge der Keimzelle und in der allgemeinen Abhängigkeit des Ganzen von der Nachbarschaft und Umwelt. Der Zellenstaat ist das Werk innerer und äußerer Anpassungserscheinungen, welche streng epigenetischen Charakter tragen und zu den Anpassungen im Freileben überleiten. 
S. 370: »Die Entwicklungsdynamik umfaßt als eine physiologische Disziplin Probleme zellenstaatlichen Wachstums und zellulärer Produktivitït, zellalärer Differenzierungsweisen in den durch ungleiches und ungleich gewordenes Wachstum, im Ringen erworbenen Situationen (Differenzierungslagen). c

Seine Lehre beruht in dieser Hinsicht also im Wesentlichen anf der Lehre von His, welche aber HAECKEL seinerzeit schroff verworfen hat; bloß wurde bei HIS nicht so sgerangen *. Diese Lehre haben auch wir Eutwicklungsmechaniker von His uibernommen, ebenso wie anderseits von HАECKEL und seinen Vorgängern deren fundamentale, damals zunächst nötige Analyse der Ontogenese in Vererbungsund Anpassungsgeschehen, welche drei Geschehensarten wir aber experimentell genauer nach Vorkommen nnd Wirken zu erforschen suchen. Unsere kansale Analyse des individuellen Entwicklungsgeschehens in: determinierende und realisierende Faktoren und in die Unterabteilungen der ersteren, sowie in >typische und atypische Entwicklung, in Neoepigenesis und Neoevolution usw. (siehe Terminologie der Entwicklungsmechanik: Analyse), ferner unsere Scheidung von Gesetzen des Wirkens und Regeln des Vorkommens, was alles zusammen die Grundlage der Entwicklnngsmechanik bildet, verwirft GREIL als überflüssig, und die mit ihrer Hilfe sexperimentells gewonnenen exakten Ergebnisse als falsch, da sie nicht zu seinen Deduktionen passen.

Es ist jedoch gut, daß Greil für »seine Art « der kausalen Ableitung auf deskriptiver Basis einen besonderen Namen, den Namen Entwicklungsdynamik, anwendet, obgleich dieser Name an sich fast dasselbe besagt wie der Name Entwicklungsmechanik. Dies erleichtert die Buchung seiner Ergebnisse. Wir werden diese später einmal zusammenstellen; glauben aber nach der ganzen Art ihrer Begrïndung und nach den bisherigen Ergebnissen, es wird mit dieser Entwicklungsdynamik gehen, wie mit dem epochalen *Konditionismus \& Verworss, welcher Terminus die VERworn eigene Bedingungslehre bezeichnen sollte, die sich aber, soweit sie wirklich etwas dem Antor Eigenes enthielt, als falsch erwies 1 ).

Die durch die kausale Analyse nötig gewordene Umdeutung des sog. biogenetischen Grundgesetzes in bloß eine Regel des sich Bewährenden habe ich juingst etwas ausführlicher begründet2). Darauf sei hier hingewiesen, da unser Autor das nicht zustimmende Urteil HaEckels iiber diese Lehre in dem seinem Buche beigelegten Reklameblatt abgedruckt hat.

Levy, Dr. Oscar, Elementares Praktikum der Entwicklungsgeschichte der Wirbeltiere, mit Einführung in die Entwicklungsmechanik. Berlin, Bornträger, 1913. $8^{0}$. 183 S. 89 Textfiguren. M. 5.60. Bibliothek für naturwissenschaftliche Praxis.

Der Verf. schildert auf Grund eigener reicher Erfahrung, wie man mit minimalen Hilfsmitteln zu Hause Beobachtungen und Versuche auf dem Gebiete der Entwicklungsgeschichte anstellen kann und lehrt, welche Versuche mit diesen

1) Siehe W. Roux, Über kausale und konditionale Weltanschauung und deren Stellung zur Entwicklungsmechanik. Leipzig, 1913. 66 S.

2) W. Roux, Die Selbstregulation, ein charakteristisches and nicht notwendig vitalistisches Vermügen aller Lebewesen. Nova Acta der Leopoldina. Bd. 100. 1914. 
Mitteln möglich sind. Zugleich werden die nötigen Vorkenntnisse in gut informierender Weise gegeben, und durch zahlreiche Textfiguren wird das Verständnis erleichtert. Den Schluß des Werkchens bildet ein Abschnitt ïber Entwicklungsmechanik, and einige Fundamentalversuche dieser neuen großen Disziplin werden mitgeteilt.

Das kleine Buch ist sehr geeignet, in weiten Kreisen von Naturfreunden die eigene Beobachtung auf dem Gebiete der beschreibenden und kausalen Entwicklnngsgeschichte zu verbreiten und so diese schwierigen Gebiete za popularisieren.

Oppel, Prof. Dr. Albert, Leitfaden für das embryologische Praktikum und Grundriß der Entwicklungslehre des Menschen und der Wirbeltiere. Jena 1914. $317 \mathrm{~S}$. u. 484 Abbildungen. M. 10.-

Der erfahrene akademische Lehrer gibt in diesem schön ausgestatteten, handlichen and billigen Buche eine sich sehr empfehlende Kombination von Theorie und Praxis, von klarer, kurzgefaßter entwicklungsgeschichtlicher Lehre und embryologischer Technik.

Zugleich ist es das erste für Studierende (der Medizin und Zoologie) und für Ärzte bestimmte embryologische Bach, welches anßer der deskriptiven Lehre auch die Grundlagen der Entwicklnngsmechanik und zwar richtig darbietet und an gutgewählten Experimenten demonstriert.

Die überaus zahlreichen Abbildungen erläutern das treffend Gesagte, so daß es leicht verständlich ist. Die Organisation des Buches ist wie die aller Bücher des Autors gut durchdacht und übersichtlich; sorgfältig angefertigte Register machen alles einzelne leicht auffindbar.

Das vorliegende Werk enthält in gedrängter Kürze und klarer Darstellung dasjenige, was zurzeit vom Mediziner an deskriptiv embryologischen und an entwicklungsmechanischen Kenntnissen verlangt werden kann und hat durch die Kombination des Beschreibenden und Kausalen den Vorzug vor allen derzeitigen embryologischen Lehrbïchern, so daß demselben weiteste Verbreitung zu wünschen ist, die durch den sehr billigen Preis erleichtert wird. In den folgenden Auflagen könnten noch weitere wichtige entwicklungsmechanische Experimente mitgeteilt werden.

Rohde, Prof. Dr. EnIL, Zelle und Gewebe in neuem Licht. Vorträge und Aufsätze äber Entwicklungsmechanik. Nr. 20. $133 \mathrm{~S}$. 40 Textfiguren. M. 5.- Leipzig, W. Engelmann, 1914.

Es entspricht dem Programm des Arehivs f. Entw.-Mech. (Bd. I, S. 24-36), » ursächliche Abhandlungen aus allen biologischen Disziplinen aufzunehmen, welche direkt ein kausal-morphologisches Ziel verfolgen and dementsprechend ihr Forschungsmaterial gesammelt und beobachtet haben $\ll$. Dies betrifft anch vergleichend-anatomische und -embryologische, also deskriptive, nicht experimentelle Arbeiten.

$\mathrm{Z} \mathfrak{u}$ diesen gehört die auf reichem vergleichenden Beobachtungsmaterial beruhende Darlegung RoHdes, in welcher er die Auffassnng vertritt, daß die Gewebezellen sehr oft nicht die direkten Abkömmlinge von Embryonalzellen, sondern 
Neubildungen sind, welche erst später durch Abgliederung aus vielkernigen Plasmamassen hervorgehen. Die biologische Differenziernng ist gleichfalls oft nicht an abgegrenzte Zellen gebunden, sondern findet in vielkernigen Plasmodien statt, und die Abgliederung der Zellen ist dementsprechend dann erst ein sekundärerer Vorgang. Dieses wird an allen Gewebsarten im einzelnen verfolgt. Der ganze Embryo würde nach der Auffassung des Verf. eine einheitliche, vielkernige Protoplasmamasse darstellen und dadurch zum Teil einem vielkernigen Protozoon entsprechen.

Die Lehre RoHdes hat in mancher Beziehung Vorläufer in Lehren von Heitzmann, Whitman, Itllie, Schlater, Heider, W. Roux u. a. und wird durch die Heranziehung der Ergebnisse der Entwicklungsmechanik sowohl Bestätigung wie auch manche Berichtigung (z. B. beziiglich der peripheren Nerven durch Harrisoss und Braus' Ergebnisse, der Selbständigkeit von Furchungszellen) erfahren. Besonders wichtig ist sie für die Differenzierung der Bindesubstanzen, zumal der Fibrillen, die danach anch unabhängig von den Zellen durch funktionelle Einwirkungen gebildet werden können. Jedenfalls verdient die Schrift RoHDes die Beriicksichtigung aller Biologen.

Triepel, Hermann, Die Ursachen der tierischen Entwicklung. Sammlung anatom. u. physiolog. Vorträge u. Aufsätze (E. GaUpP u. W. Trendelenburg). 20. Heft. Jena, G. Fischer, 1913. $47 \mathrm{~S}$.

Der Antor legt seine Stellung zu vielen Ergebnissen der entwicklungsmechanischen Forschung dar. Er klassifiziert zunächst die verschiedenen Ursachen biologischen Geschehens in zwei Hauptgruppen. Der Biologie selber entnommene Ursachen will er homotrope, die der Physik und Chemie entstammenden allotrope Ursachen nennen. Ich bezweifle indes, daß diese Scheidung und Benennung nötig oder nützlich ist, denn meine bereits eingebürgerte Distinktion von einfachen und komplexen Faktoren besagt im Grunde dasselbe. Auf die beiden Gruppen sucht er die kausalen Ergebnisse zu verteilen. Die Darstellung ist klar und darch viele Beispiele illustriert. Manche seiner Gedanken sind weiterer Beachtung und Prüfung wert; manches kann ich dagegen schon ietzt als nicht zutreffend bezeichnen. Einiges davon sei hier gleich erwähnt:

Triepel sagt (S. 6; : ,Eine Ursache kann immer nur eine Wirkung haben «. Demgegenüber sei darauf hingewiesen, daß nie eine Ursache etwas wirkt, jedes Geschehen hat wenigstens zwei Ursachen 1). Ferner sagt der Autor (S. 29): -Wenn jemand eine Struktur als funktionell bezeichnet, so sagt er uns eigentlich nichts Nenes «. Wenn aber diese »der Funktion hochgradig angepaßte "Beschaffenheit vorher noch nicht als solche erkannt war, so ist die Aussage doch etwas Nenes. Nur prinzipiell ist solche Ermittlang jetzt (!) nichts Neues mehr, da wir schon viele solche Strukturen kennen.

S. 33 äußert sich Trieper: 2 Mir will es scheinen, als ob durch die Einführung des Begriffes ,trophischer Reiz' für die Erklärung der funktionellen Strukturen nicht viel gewonnen wäre. Er ist kaum mehr als eine Umschreibung der erschlossenen Vorgänge oder, wenn man will, eine näbere Präzisierung «. Wesentlich dieselbe Ansicht hat bereits DrIESCH (1899) geäußert.

1) Siehe hierzu auch: Rovx, Über kausale und konditionale Weltanschauung und deren Stellung zur Entwicklungsmechanik. Leipzig 1913. 
Beide Autoren ahnen offenbar nicht, was sie einerseits mir damit für eine Anerkennung aussprechen, indem sie meine Erklärung der Entstehung von Millionen verschiedenen »zweckmäßigen neuen Gestaltungen in neuen Verhältnissen als nur eine Umschreibung der erschlossenen Vorgänge oder als eine Photographie des Problems (DrIEsch) bezeichnen, denn sie erkennen damit diese Erklärung als die richtige und als sicher an. KAssowirz dagegen hat (1908) bezüglich der Knochen (unter Übergehung meiner Lehre) eine wesentlich andere Ableitung gegeben. Anderseits uibergehen sie aber das Historische der Sache; sie erinnern sich nicht mehr daran, daß es nach Ermittlung der Tatsachen der funktionellen Anpassung eine Zeit gab, in der die Forscher glaubten, diese wunderbar zweckmäßigen Tatsachen wären nur durch eine zwecktätig gestaltende Intelligenz möglich. Mein Nachweis, daß letztere Annahme nicht nötig ist, sondern daß diese Struktaren entstehen müssen, sofern nur der funktionelle Reiz zugleich trophische Wirkung hat, war damals, wie mir LeUKART 1876 sagte, eine Erlösung, eine Befreiung. Jetzt hinterher, nachdem wir im Besitze dieser mechanistischen Erklärung sind, werden die bezüglichen Tatsachen einfach sals selbstverständliche in das Fach der Reizwirkungen getan.

TRIEPEL will (S.12) die ganze individuelle Entwicklnng in folgende drei Perioden teilen: ${ }_{D}$ Die erste Periode ist die der Selbstdifferenzierung; in der zweiten kommt zu der unabhängigen noch abhängige Differenzierung hinzu; diese ist in der dritten Periode allein wirksam «. Diese Einteilung ist indes prinzipiell unzutreffend, da auch die Selbstdifferenziernng von Gebilden nicht in lauter Selbstdifferenzierung der Unterteile des Gebildes besteht und bestehen kann; dies deshalb, weil in letzter Instanz alle Selbstdifferenzierung von Gebilden oder Teilen nur durch differenzierende Wirkungen von Unterteilen aufeinander, also durch abhängige Differenzierung geschehen kann. Selbstdifferenziernng bezeichnet keine Art des Geschehens, sondern bloß den Sitz der determinierenden Faktoren des Geschehens in dem betreffenden Gebilde. Deshalb ist Triepels Einteilung der Ontogenese durchaus unmöglich. Ich habe als erste Periode die der vererbten Differenzierung, also ohne Wirkung des funktionellen Reizes, bezeichnet; und in dieser Periode kommt, wie ich u. A. erwiesen haben, viele Selbstdifferenzierung von Teilen vor, die in meiner dritten Periode fehlt. Auf diese Weise richtig bezeichnet, wäre Triepels Einteilung mit der meinigen identisch, besagte aber nichts Neues.

Der Autor hat Vieles durchdacht und sich auf eigene Weise zurechtgelegt. Das sei als verdienstlich anerkannt, wenn auch manchen seiner Denkergebnisse nicht zuzustimmen ist; das betrifft besonders auch diejenigen über die Theorie der funktionellen Anpassung, die von mehreren Autoren sehr unrichtig beurteilt worden ist, seitdem Driesch sie seinerzeit (Anatomische Hefte 1899) in einem im übrigen sehr verdienstlichen Referat wesentlich unrichtig dargestellt hat.

Roux.

voN UexküLL, J., Bausteine zu einer biologischen Weltanschaung. Gesammelte Anfsätze. Herausgegeben und eingeleitet von FeLIX Gross. München 1913. $298 \mathrm{~S}$.

Der Herausgeber des Buches versteht unter »Biologie» befremdlicher Weise nur die Lehre von den äußeren Lebensumständen der Lebewesen und bezeichnet den Autor, der sich experimentell mit der Erforschung der Wirkungen dieser änßeren Umstände auf die Lebewesen beschäftigte, nachdem dies früher 
zumeist ohne Experiment gesehehen ist, als den Begründer der modernen experimentellen Biologie in Deutschland. Weiß Gross nicht, daß Biologie die gesamte Lebenslehre bedeutet, und $\mathrm{da} B$ die Physiologie, die Entwicklungsmechanik und die Pathologie seit lange schon sexperimentelle Biologies darstellen? v. UEXKÜLL gehört zu jener Gruppe von Autoren, für welche die rein kausalen biologischen Disziplinen, mit ihnen auch der Darwinismus, widerlegt sind.

Wir wollen hier von dem, mit der vollen Überzeugung des absolut Richtigen Niedergeschriebenen und daher anf die große Menge der Unselbständigen überzeugend wirkenden theoretisehen Inhalt des Buches nur auf das gegen die Entwicklungsmechanik gerichtete, auf willkiurlicher Deutung einiger ibrer Ergebnisse beruhende Resultat seines Denkens hinweisen (S. 270-273).

»Der noch nicht entwickelte Keim hat keine Struktar.s "Die Strukturbildung ist ein unabhängiger Naturfaktor, der keine Struktur ist. « Neben Materie, Energie, Struktar, tritt als vierter Naturfaktor der Strukturbildner.s ১Der Strukturbildner ist unabbängig von der Struktur und daher unzerstörbar, ewig.« $D$ Der Strukturbildner gehorcht nicht dem Kausalgesetz, sondern schreibt Gesetze vor, die wir zweckmäßig nennen.

Wir kausalen Forscher billigen diese iberaus bequeme, aber auch nichts erklärende Methode der "Erklärang nicht. Ich verweise in dieser Hinsicht auf meine neueste Schrift: Die Selbstregulation, ein charakteristisches und nicht notwendig vitalistisches Vermögen aller Lebewesen. Nova Acta der Leopoldina. Bd. 100. 1914.

Für uns baben daher bloß die experimentellen Untersuchungen des Autors Wert. 